\title{
R egeneración post-incendio del ciprés de la cordillera (Austrocedrus chilensis) en los primeros años
}

\author{
First years of postfire regeneration in cypress forests (Austrocedrus chilensis) \\ C.D.0.: 434 \\ MIRIAM GOBBI, LUIS SANCHOLUZ \\ Depto. de Ecología, Centro Regional Universitario Bariloche, Universidad \\ del Comahue, c.c. 1336, San Carlos de Bariloche, A rgentina.
}

\begin{abstract}
SUM M ARY
Due to a series of biological and ecological characteristics, andean cypress forests have been and continue to be frequently affected by forest fires. This study provides a first evaluation of post-fire regeneration in pure cypress forests which were burnt up to fifteen years ago. To evaluate post-fire succession, plant cover by strata and cypress abundance were studied. Experiments were also conducted on field and laboratory germination with soils with different burning ages, under different watering regimes in the laboratory. The seed bank is also studied. Field results show a lack of regeneration in all burnt soils. Field and laboratory experiments provide no evidence of the effects of soil burning on germination rates. The poverty of the seed bank in the burned soils may explain the lack of regeneration.
\end{abstract}

\section{RESUMEN}

Los bosques de ciprés de la cordillera, debido a una serie de características biológicas y ecológicas, han sido y son frecuentemente afectados por los incendios forestales. En este trabajo se ofrece una primera evaluación de la regeneración post-incendio en bosques puros de ciprés, incendiados hasta quince años atrás. Para evaluar la sucesión post-incendio se analizan la cobertura de los distintos estratos de la vegetación y la abundancia del ciprés. También se estudia la germinación en campo y en laboratorio en suelos con distintas edades de incendio, considerando diferentes niveles de riego en laboratorio. Para evaluar el efecto de la dispersión de los propágulos se analiza el banco de semillas de ciprés. Los resultados indican ausencia de regeneración en las áreas quemadas. De los experimentos se concluye que los posibles cambios del suelo no afectarían a la regeneración. En cambio, la pobreza del banco de semillas en los suelos quemados se explicaría por la falta de regeneración.

\section{INTRODUCCION}

Los incendios forestales han sido la principal causa de alteración en los bosques andino-patagónicos (Rothkugel, 1913, 1916; Solbrig, 1984; B ailey Willis, 1988). El ciprés de la cordillera (Austrocedrus chilensis) forma parte de estos bosques entre los $36^{\circ} 30^{\prime}$ y los $43^{\circ} 55^{\prime}$ (Hueck, 1978) y sobre su distribución natural se sobrepone la mayor actividad humana en la norpatagonia argentina (Dezzotti, 1989). Esta coincidencia espacial de los cipresales con los asentamientos humanos crea áreas de alto riesgo de incendio (Bruno, 1982).
El ciprés se quema en forma rápida, probablemente debido a que el contenido resinoso de su follaje arde explosivamente (Donoso, 1981). Hay evidencia de que el ciprés recoloniza, a largo plazo, las áreas incendiadas (Lebedeff, 1942; D onoso, 1981; Seibert, 1982 y Veblen y Lorenz, 1987, 1988). No hay evidencias de que se reproduzca vegetativamente y poco se sabe sobre los procesos sucesionales post-incendio.

En los bosques quemados se producen cambios, como el incremento en la intensidad lumínica que resulta de la destrucción de árboles, cambios en la humedad, nutrientes y textura de los suelos (K ozlowski y A hlgren, 1974). A demás, en la capa 
superficial del suelo, donde las nuevas raíces comienzan a establecerse, los períodos de calor son más largos y más anchas las fluctuaciones de temperatura y humedad (A hlgren, 1974).

Los reportes sobre el efecto de la ceniza en la germinación y la implantación radical son contradictorios. A hlgren (1974), citando a otros autores, da ejemplos donde la germinación en pinos se ve inhibida, no se ve afectada, o bien se ve estimulada, pero se inhibe la penetración radicular.

Los efectos de los incendios forestales resultan altamente variables como consecuencia de la intensidad, frecuencia, profundidad y época del año del incendio, así como de diferencias climáticas, del tipo de suelo, de la capacidad de las especies para rebrotar y/o almacenar semillas y del espesor de la corteza de los árboles en cuestión (Little, 1974).

El ciprés es una especie arbórea de gran porte, que produce abundantes semillas livianas, que habita en zonas ventosas, que tolera condiciones xéricas y que prospera en suelos poco profundos. Con estas características es esperable que el ciprés recolonizara prontamente las zonas quemadas.

Una pregunta surge a la luz de la teoría sucesional moderna (Pickett et al., 1987): ¿difiere el ciprés en su disponibilidad para colonizar las zonas quemadas o bien presenta un comportamiento diferencial en dichas zonas?

Este trabajo ofrece una primera evaluación de la regeneración post-incendio de bosques puros de ciprés quemados hasta quince años atrás. Para ello: i) se caracterizan los estadios sucesionales de la vegetación, ii) se analizan, en particular, las influencias del suelo, y del agua sobre la germinación y la implantación de radículas, y iii) se evalúa a través del banco de semillas la dispersión de propágulos.

\section{MATERIALES Y METODOS}

En 1990, y a partir de registros de incendios de la Dirección Forestal Andina de la Provincia de Río Negro y de la Dirección de Parques $N$ acionales (A rgentina), se seleccionaron tres sitios con bosque puro de ciprés en los que se hubiesen quemado más de 10 há en los últimos quince años en las proximidades de San Carlos de Bariloche: V entana, Piscicultura y Catedral. La localización de los mismos fue corroborada por pobladores del lugar. Los dos primeros sitios se incendiaron en 1987 y el último en 1976, todos en verano. La intensidad de estos incendios debe haber sido alta, ya que, a excepción de un ciprés adulto, la vegetación fue totalmente quemada. Piscicultura y Ventana difieren en el tipo de bosque, el primero es más húmedo y en él participa el coigüe (Nothofagus dombeyi) en el dosel.

En cada uno de estos sitios se identificaron tres zonas de estudio que corresponden con el grado de incidencia del incendio:

a) Quemado (q) es la zona más afectada por el incendio; hay pocos árboles sobrevivientes y éstos han perdido por ignición sus ramas más pequeñas y bajas y los troncos tienen sectores carbonizados; gran parte de los árboles muertos están caídos.

b) Borde (b) es la zona donde hay claros signos de incendio, pero no hay árboles caídos o son muy raros; la mayoría de los árboles no ha muerto, aunque presenten algunos signos de daño por fuego y conserven parte, cuando no todo, del follaje.

c) Testigo (t) a la zona que no presenta signos visibles de fuego.

En cada una de las zonas descritas se delimitaron aleatoriamente seis unidades muestrales cuadradas de $16 \mathrm{~m}^{2}$, en las que se evaluaron: porcentaje de suelo descubierto, de cobertura por estrato de la vegetación y numerosidad del ciprés en las siguientes clases de tamaño: a) plántula: con hojas embrionarias; b) plantín: con hojas diferenciadas y menos de $10 \mathrm{~cm}$ de altura; c) juvenil: mayor de $10 \mathrm{~cm}$ de altura y menor de $10 \mathrm{~cm}$ de diámetro a la altura del pecho (DAP) y d) adultos: mayor de 10 $\mathrm{cm}$ de DAP.

A fines de 1990 se iniciaron ensayos de laboratorio para evaluar, por un lado, y en forma preliminar, el efecto de los suelos quemados sobre la germinación $y$, por otro, la implantación de radículas de ciprés. Un año después se analizó el efecto de distintos riegos sobre la germinación, según el suelo fuese quemado o no. Para ello se contó con semillas recogidas en abril del año del ensayo en las proximidades de San Carlos de Bariloche. Estas semillas fueron seleccionadas una a una a fin de descartar rotas y fallidas. Se estratificaron a $4-5^{\circ} \mathrm{C}$ en arena húmeda durante 60 días. Las muestras de suelo para los ensayos fueron tomadas cuidando de no alterar la estructura superficial del suelo; se hicieron seis repeticiones de 
cada tratamiento que se colocaron en cajas plásticas de $10 \times 15 \times 3 \mathrm{~cm}$ de profundidad a temperatura ambiente.

Las semillas de ciprés son chicas y livianas, con un peso aproximado de $4 \mathrm{mg}$ y una longitud, incluida el ala, menor a $10 \mathrm{~mm}$.

Para los ensayos de germinación en laboratorio se sembraron en cada caja 50 semillas a aproximadamente $0.5 \mathrm{~cm}$ de profundidad. La emergencia de plántulas se contó hasta 50 días después de sembradas. Los niveles de riego fueron equivalentes a 2, 3.5 y $4.7 \mathrm{~cm} / \mathrm{mes}$ y se trabajó con muestras de suelo quemados y testigos provenientes de Piscicultura y de Catedral. Se pusieron a germinar a igual régimen de riego muestras de suelo, sin siembra, tomadas colindantes a las utilizadas para germinación, a fin de cuantificar el potencial germinativo del suelo utilizado y descartar la posible influencia de la semilla del banco en los ensayos de laboratorio.

El ensayo de implantación de radículas se realizó con semillas germinadas en humedad sobre algodón en cajas de plástico. Cuando las radículas medían entre 2 y $15 \mathrm{~mm}$ fueron colocadas en cajas con suelos provenientes del testigo y del quemado de Piscicultura. Se contó la emergencia de las plántulas durante 45 días.

En el campo se comparó la germinación en las tres zonas ( $a, b$ y $t$ ) de los sitios Piscicultura y Catedral. En octubre de 1990 se sembraron 50 semillas, previamente estratificadas, en cuadrados de $20 \times 20 \mathrm{~cm}$, cubriéndolas con $0.5 \mathrm{~cm}$ de tierra. En cada uno de estos cuadrados se registró la cobertura herbácea, arbustiva y arbórea y la pendiente en una escal a relativa de 0 a 4 . En cada zona se realizaron 10 repeticiones. Se contaron plántulas emergidas durante ese verano y el siguiente.

Para evaluar el banco de semillas de ciprés se tomaron en total 36 muestras de $150 \mathrm{~cm}^{2}$ hasta 2-3 $\mathrm{cm}$ de profundidad en las zonas testigo, borde y quemado, tanto de Piscicultura como de Catedral. Las muestras de la zona quemada se tomaron a partir de $10 \mathrm{~m}$ del borde del incendio y en una franja de $20 \mathrm{~m}$ de ancho. Cada muestra se pasó por un cedazo de malla fina, a fin de eliminar la tierra y luego se separaron con una pinza y se contaron todas las semillas. Se consideró como una semilla todas aquellas en las que por lo menos quedara la mitad de una entera. Además en Piscicultura se midió la abundancia del banco de semillas en el quemado a distintas distancias del borde (entre 10 y $30 \mathrm{~m}$ ).
El diseño utilizado corresponde en todos los casos a uno completamente aleatorizado. Los resultados de los ensayos de germinación en laboratorio con distintos niveles de riego se analizaron mediante un ANOVA a dos factores: riegos ( $3 \mathrm{ni}-$ veles) y tipo de suelo (4 niveles) con la variable transformada $\left(\mathrm{y}^{\prime}=\right.$ número de semillas germinadas). Con un A NOVA a un factor se analizaron los resultados comparando dos suelos quemados con igual antigüedad ( 3 años) de distintos sitios (Piscicultura y Ventana), la implantación de radículas y el banco de semillas con la variable transformada a $y^{\prime}=$ In (número de semillas +1 ).

En todos estos ensayos se probó normalidad y homoscedasticidad y se efectuaron comparaciones múltiples a posterior cuando correspondieron (Test de Tukey). Los resultados de las germinaciones en campo se analizaron mediante el Test de K ruskalWallis. Los niveles de significancia utilizados para rechazar las hipótesis fueron del $5 \%$.

\section{RESULTADOS}

a) Descripción física y de la vegetación de los sitios. No existen registros precisos de precipitación para los sitios estudiados, una aproximación se puede obtener del mapa de Barros et al. (1979), que indica para V entana 1.400 , para Piscicultura 1.600 y para Catedral $1.800 \mathrm{~mm}$ anuales. La pendiente es similar en los tres sitios, variando entre 21 y $27^{\circ}$. En las numerosas visitas a los sitios de estudio no se pudo observar ganado ni rastros del mismo (ramoneo, pisoteo y bosteo). La actividad ganadera en estos sitios periurbanos es prácticamente nula.

En el cuadro 1 se muestra el porcentaje de suelo descubierto y la cobertura porcentual por estrato para las tres zonas de cada sitio. El suelo descubierto alcanza su valor máximo en la zona quemada de Ventana. El estrato arbóreo no se ha desarrollado en ninguna de las zonas quemadas. El estrato arbustivo está ausente en la zona quemada de Ventana y alcanza su máximo desarrollo en la zona quemada de Catedral $(71.5 \%)$, que presenta una fisonomía de matorral al to con especies que llegan a los $3 \mathrm{~m}$ de altura. El estrato herbáceo muestra una pronta recuperación.

b) Regeneración del ciprés en las zonas y sitios. El cuadro 1 muestra, en sus últimas filas, los resultados de abundancia de ciprés por clases de tamaño y evidencian la ausencia de regeneración del mismo en las zonas quemadas de los sitios 


\section{CUADRO 1}

\section{Suelo descubierto (\%), Cobertura por estrato $(\%)$ y A bundancia de ciprés ( $N^{\circ}$ ind/há) para las distintas zonas y sitios relevados}

Bare soil (\%), Plant cover by strata (\%) and cypress abundance (№ ind/ha) for the different zones and sites studied

\begin{tabular}{|c|c|c|c|c|c|c|c|c|c|}
\hline \multirow{2}{*}{$\begin{array}{l}\text { Sitio } \\
\text { Zona }\end{array}$} & \multicolumn{3}{|c|}{ Ventana } & \multicolumn{3}{|c|}{ Piscicultura } & \multicolumn{3}{|c|}{ Catedral } \\
\hline & Testigo & Borde & $\begin{array}{l}\text { Que- } \\
\text { mado }\end{array}$ & Testigo & Borde & $\begin{array}{l}\text { Que- } \\
\text { mado }\end{array}$ & Testigo & Borde & $\begin{array}{l}\text { Que- } \\
\text { mado }\end{array}$ \\
\hline$\%$ Suelo descubierto & 51.7 & 28.3 & 89.7 & 37.5 & 53.3 & 48.8 & 25.8 & 50.8 & 35.0 \\
\hline $\begin{array}{l}\text { \% Cobertura } \\
\text { Arbórea } \\
\text { Arbustiva } \\
\text { Herbácea }\end{array}$ & $\begin{array}{l}65.8 \\
10.3 \\
10.7\end{array}$ & $\begin{array}{l}43.3 \\
28.1 \\
25.2\end{array}$ & $\begin{array}{r}0.0 \\
0.0 \\
12.3\end{array}$ & $\begin{array}{l}80.0 \\
72.9 \\
14.2\end{array}$ & $\begin{array}{l}45.0 \\
22.0 \\
14.8\end{array}$ & $\begin{array}{r}0.0 \\
23.1 \\
16.5\end{array}$ & $\begin{array}{l}61.7 \\
23.5 \\
16.3\end{array}$ & $\begin{array}{l}61.0 \\
23.5 \\
18.9\end{array}$ & $\begin{array}{r}0.0 \\
71.5 \\
10.9\end{array}$ \\
\hline $\begin{array}{l}\text { A bundancia (№/há) } \\
\text { Adultos } \\
\text { R enovales } \\
\text { J uveniles } \\
\text { (> } 150 \mathrm{~cm} \text { altura) } \\
(50-149 \mathrm{~cm} \text { altura) } \\
(10-49 \mathrm{~cm} \text { altura) } \\
\text { Plantines } \\
\text { Plántulas } \\
\text { Test de abundancia entre } \\
\text { zonas testigo y borde }\end{array}$ & $\begin{array}{r}437.5 \\
0.0 \\
187.5 \\
0.0 \\
0.0 \\
\end{array}$ & $\begin{array}{r}437.5 \\
125.0 \\
312.5 \\
0.0 \\
1.125 .0 \\
10)=0 .\end{array}$ & $\begin{array}{l}0.0 \\
0.0 \\
0.0 \\
0.0 \\
0.0\end{array}$ & $\begin{array}{r}3.125 .0 \\
1.875 .0 \\
500.0 \\
187.5 \\
0.0 \\
\\
F(1 .\end{array}$ & $\begin{array}{r}1.062 .5 \\
437.5 \\
187.5 \\
187.5 \\
0.0 \\
\\
\\
0)=2.3\end{array}$ & $\begin{array}{l}0.0 \\
0.0 \\
0.0 \\
0.0 \\
0.0\end{array}$ & $\begin{array}{r}1.250 .0 \\
625.0 \\
812.5 \\
375.0 \\
937.5 \\
\text { F (1. }\end{array}$ & $\begin{array}{r}625.0 \\
437.5 \\
125.0 \\
437.5 \\
0.0\end{array}$ & $\begin{array}{l}0.0 \\
0.0 \\
0.0 \\
0.0 \\
0.0\end{array}$ \\
\hline
\end{tabular}

estudiados, no así en las zonas borde y testigo, que no presentan diferencias significativas. No aparecen renovales, aun en lugares con una alta cobertura arbustiva, como es el caso ya mencionado de la zona quemada de Catedral, en el que habían pasado 14 años al momento del relevamiento desde el incendio. Todos los renovales encontrados en recorridas aleatorias del área se hallaban próximos a ejemplares adultos remanentes del incendio.

c) Germinación en laboratorio. La figura 1 muestra los resultados de la germinación acumulada (\%) en 50 días para los distintos niveles de riego en suelos testigos y quemados de Piscicultura y Catedral. La interacción resultó significativa $\left(\mathrm{F}_{6.60}=7.49\right)$. Cuando se analizan los resultados por sitios, resulta significativa la interacción entre riegos y zonas en Piscicultura $\left(F_{2.30}=5.83\right)$, pero no así en Catedral. En este último sitio el efecto zona no es significativo $\left(F_{1.30}=4.14\right)$, pero sí lo es el efecto riego $\left(F_{2.30}=28.37\right)$. Este último resultado es coincidente con el de experiencias preliminares, en las que se compararon suelos de zonas quemadas de Piscicultura, Ventana y Catedral contra sólo la zona testigo de Piscicultura, con riego suficiente para mantener todos los tratamientos continuamente húmedos. En estas experiencias también se encontró que la germinación es similar en zonas quemadas y zonas testigo $\left(F_{2.21}\right.$ $=0.60)$, independientemente del tiempo transcurrido desde el incendio.

d) Germinación en el campo. Se observó una bajísima germinación en el primer verano posterior a la siembra (1991), con valores de $0.2 \%$ en borde y testigo de Piscicultura y $0 \%$ en las demás zonas consideradas. Un año después, verano de 1992, los porcentajes de germinación aumentaron, salvo en la zona quemada de Piscicultura (figura 2). Los valores máximos, 4.7 y $8.4 \%$, coinciden con las zonas de mayor cobertura arbustiva, quemado de Catedral y testigo de Piscicultura, respectivamente. Coincidentemente las unidades muestrales con mayor porcentaje de germinación están bajo la protección de arbustos donde habría mayor humedad.

e) Implantación de radículas. En el ensayo de implantación de radículas se llega a resultados similares a los obtenidos para los ensayos de germinación, ya que no hay diferencias significativas al final del ensayo $\left(F_{1.10}=0.01\right)$ con medias de 81 


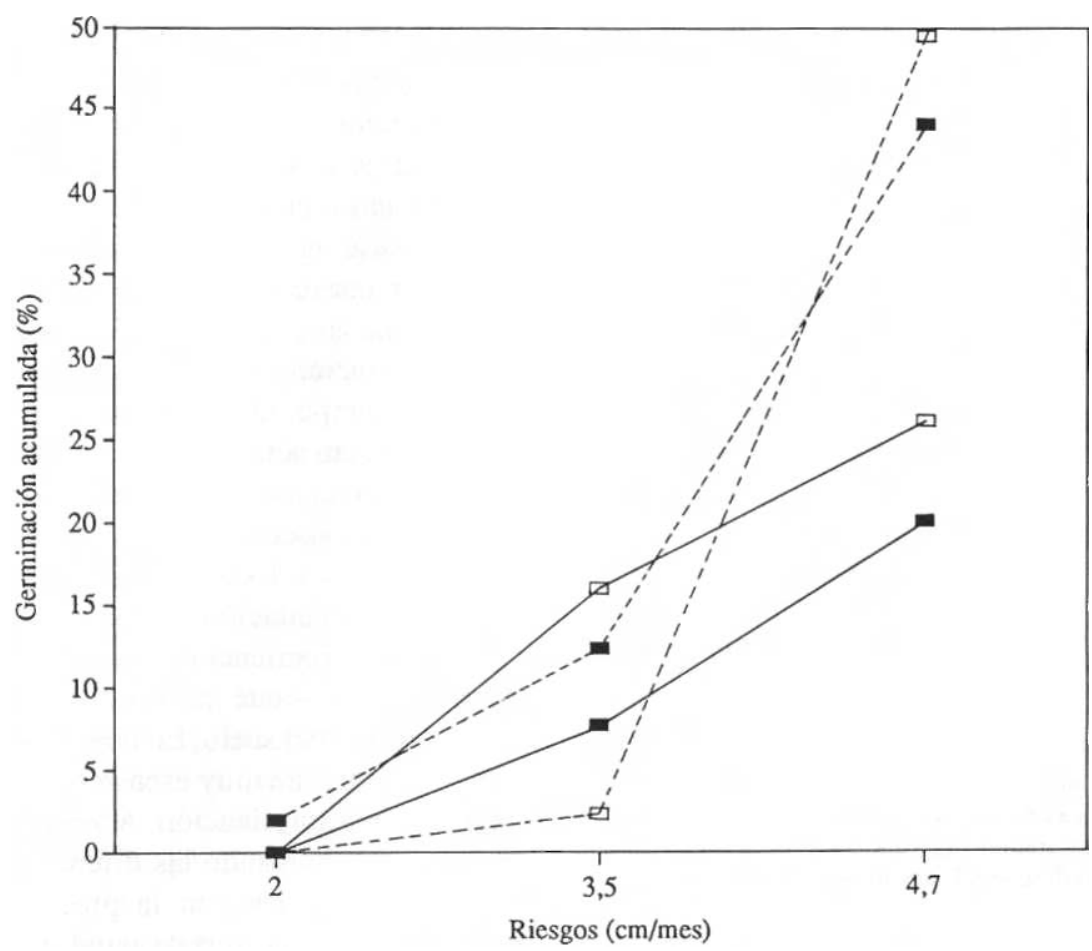

Figura 1. Germinación acumulada (\%) en 50 días para distintos niveles de riego $(2,3.5$ y $4.7 \mathrm{~cm} / \mathrm{mes})$, para las zonas (ㅁ: testigo; $\square$; quemado) y para los sitios (--: Piscicultura; : Catedral).

Cumulative geimination (\%) in 50 days for different watering levels (2, 3.5 and $4.7 \mathrm{~cm} /$ months), for zones (- control; $\square$ : burnt) and for sites (---: Piscicultura; : Catedral).

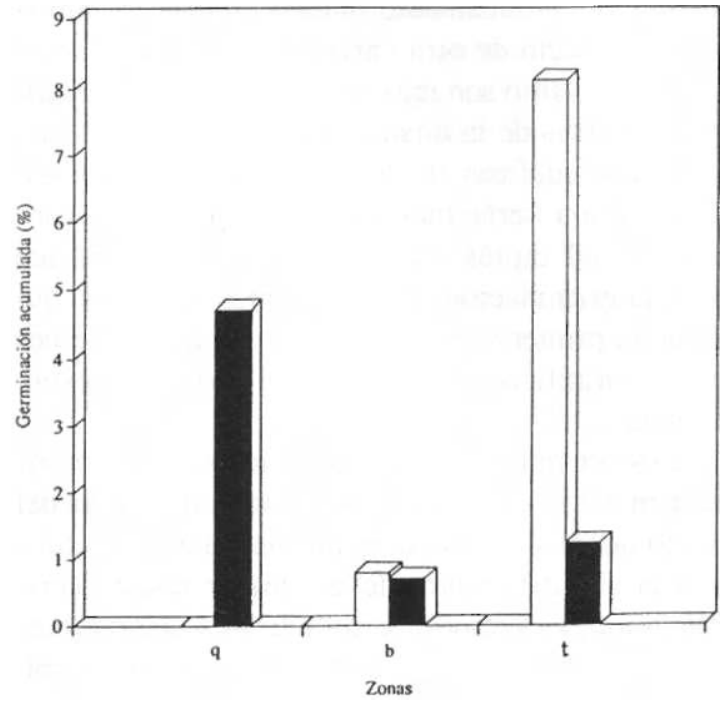

Figura 2. Germinación acumulada de 1992, calculada como \% de semilla entera sembrada en campo para las tres zonas (q: quemado; b: borde y t: testigo) en Piscicultura ( $\square$ ) y Catedral ( $\square$ ).

Cumulative germination as $\%$ of whole seeds sown in the field for the three study zones ( $q$ : burnt; $b$ : border and t: control) in Piscicultura ( $\square$ ) and Catedral ( y $81.4 \%$ para suelos con 5 años de quemado y su testigo, respectivamente.

f) Banco de semillas. Los resultados del banco de semillas en distintas zonas se muestran en la figura 3. En los dos sitios considerados la abundancia de semillas en el banco del suelo decae bruscamente hacia la zona quemada, aun a pocos metros del borde. En el banco de los suelos quemados hay sólo un 2.4 y un $4.1 \%$ de las semillas de los suelos testigos de Catedral y Piscicultura, respectivamente. Los resultados del A NOVA para Piscicultura indican dos grupos con medias significativamente diferentes $\left(F_{2.15}=10.93\right)$ : quemado $<$ testigo = borde; para Catedral los grupos formados son tres: quemado < borde < testigo. La dirección dominante de los vientos varía de un sitio a otro: en Catedral los vientos soplan hacia el testigo, al revés de lo que ocurre en Piscicultura.

\section{DISCUSION}

A I evaluar la regeneración natural en las áreas quemadas comprendidas en este estudio se nota la 


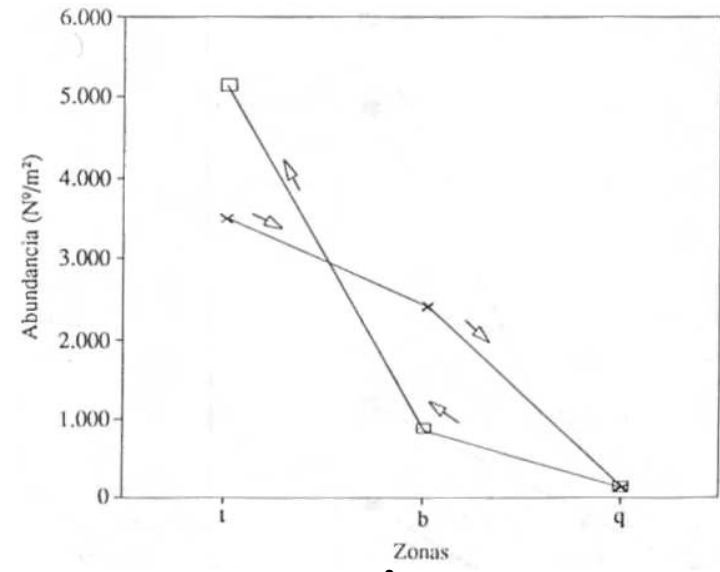

Figura 3. Abundancia $\left(\mathrm{N}^{\circ} / \mathrm{m}^{2}\right)$ de semillas de ciprés en el banco para las zonas (t: testigo; b: borde y q: quemado). En Piscicultura (X) $\mathbf{q}<\mathbf{b}=\mathbf{t}$, con $F_{2.15}=10.929$; en Catedral ( $\square$ ) $\mathbf{q}<\mathbf{b}<\mathbf{t}$ con $\mathbf{F}_{2.15}=36.642$. L a dirección predominante de los vientos se indica mediante flechas. Cypress seed abundance in the seed bank $\left(\mathrm{N}^{\circ} / \mathrm{m}^{2}\right)$ for zones $(\mathrm{t}$ : control; b: border and q: burnt). In Piscicultura (X) $\mathrm{q}<\mathrm{b}=\mathrm{t}$, $\mathrm{F}_{2.15}=10.929$; in Catedral $(-) \mathrm{q}<\mathrm{b}<\mathrm{t}, \mathrm{F}^{2} .25=36.642$. The direction of prevailing winds are shown with arrows.

ausencia de renovales y de rebrotes de cipreses. Esto no puede ser atribuido a la ausencia de cobertura vegetal, que eventualmente evitaría la desecación y daría sombra y protección a los renovales, ya que se ha analizado una serie de sitios con distintos niveles de cobertura y en ningún caso se observan individuos de la especie en estudio. Como consecuencia de las observaciones de campo, respecto a la presencia y efecto del ganado, descartamos la influencia del pastoreo en nuestros resultados de regeneración.

L a mayoría de las especies del estrato herbáceo y todas las del arbustivo del testigo estaban presentes en el área quemada más antigua (Catedral), salvo el ciprés.

Estos resultados difieren de los citados por López et al. (1988) para un bosque de ciprés con un año de quemado: encontró una regeneración natural evaluada a través de la cantidad de semilla germinada igual a 4.500 plántulas/ha. Es importante destacar que en el mismo sitio sobrevivió al incendio el $44.5 \%$ de los cipreses.

Como ya se mencionó en la introducción, el ciprés puede, a largo plazo, recolonizar áreas quemadas. Para su germinación y supervivencia temprana en vivero se ha notado la importancia de la sombra artificial para evitar la desecación (L ebedeff, 1942; Rovere, 1991) y, en campo,
Lebedeff (1942) menciona la aparición de renovales bajo arbustos, sin definir cuál de los siguientes sería el factor responsable: protección contra los rayos solares, eliminación del efecto sofocante de las herbáceas o bien protección contra animales, a lo que podría agregársele conservación de la humedad. En nuestro trabajo podríamos esperar, por lo tanto, que las condiciones fueran propicias para la recolonización, al menos en la zona quemada hace más tiempo. Q ue esto no ocurra permite suponer que están actuando otros factores. L as posibles transformaciones edáficas que ocurrirían en el suelo, como consecuencia del paso del fuego, tampoco parecen ser decisivas, ya que ni la germinación ni la implantación de radículas fueron diferentes en las experiencias realizadas.

Parece interesante profundizar en el análisis de la humedad del suelo. En nuestras experiencias, cuando el agua fue muy escasa, se notó efecto del fuego sobre la germinación. A valores intermedios de riego, que, salvando las diferencias campo-laboratorio, coinciden con la precipitación media para el verano en la zona de estudio, el único suelo que difiere de los restantes es el quemado más reciente (Piscicultura), el único en que no se verificó germinación de las semillas sembradas en la experiencia en campo. Cuando el agua dejó de ser un factor limitante $(4.7 \mathrm{~cm} / \mathrm{mes})$ y el suelo se mantuvo continuamente húmedo parece manifestarse el efecto de otra variable, ya que los suelos del mismo sitio son más parecidos entre sí que los provenientes de la misma zona. Quizás las características edáficas de los sitios sean diferentes: Piscicultura sería más favorable para la germinación del ciprés que Catedral. Probablemente la baja germinación de las siembras en campo durante el primer verano se deban a imperfecciones en la estratificación artificial utilizada en la experiencia.

Los porcentajes de germinación de campo ponen en evidencia la al ta capacidad germinativa del suelo quemado más antiguamente que no se diferencia significativamente del mejor testigo (Piscicultura), y es muy superior a los resultados obtenidos por Rovere (1991) con estratificación natural.

El descenso brusco del banco de semillas de cipreses hacia las zonas quemadas impediría la recolonización. En estas condiciones la aparición de cipreses en las zonas quemadas dependería de un avance desde la zona de bordes, donde la regeneración es similar a la del testigo, o bien de algún ejemplar sobreviviente al incendio, y estaría in- 
fluida por el sentido de los vientos, y, eventualmente, por la pendiente. Esto explicaría por qué la zona de Catedral, con vientos que soplan desde el quemado hacia el testigo, tiene una relación de bancos de semilla borde/testigo menor que la de Piscicultura, con vientos que soplan en sentido contrario. De cualquier manera este efecto parece ocurrir en pequeñas distancias, ya que, ni aun a favor del viento y luego de cinco años de liberación de semillas, el banco de semillas en la zona quemada de Piscicultura alcanza un tamaño considerable.

\section{CONCLUSIONES}

No se ha verificado regeneración del ciprés en las zonas recientemente quemadas, ni por germinación de semillas ni por rebrote, independientemente de los distintos grados de cobertura vegetal.

$\mathrm{No}$ hay evidencias que indiquen que los suelos quemados impidan la germinación ni la implantación de radículas de ciprés.

La humedad parece ser una variable importante, pero la ausencia de semillas en las zonas quemadas, aun a poca distancia de los adultos del borde, parece ser la variable decisiva en la ausencia de regeneración del ciprés.

Si se sorteara la dificultad de la ausencia de semillas, otras variables manifestarían su importancia. Quizás, entonces, un aumento de la humedad, que podría ser consecuencia del aumento de cobertura vegetal que se produce en los incendios más antiguos sería el mecanismo que explica la recolonización observada en zonas con incendios muy antiguos (mayores de 50 años). Si éste fuese el camino la sucesión en bosques de ciprés seguiría el camino de la facilitación (Connell y Slatyer, 1977).

Considerando las causas generales de la sucesión propuesta por Picket et al. (1987), en este trabajo se concluye que la disponibilidad del ciprés para recolonizar es diferente en las zonas quemadas, por reducción del banco de semillas, y no se han hallado evidencias de que presente un comportamiento diferencial ni en la germinación ni en la implantación de radículas.

\section{A GRADECIMIENTOS}

Los autores quieren expresar su agradecimiento al técnico $\mathrm{M}$ arcelo A lonso por su asistencia en los muestreos, a las licenciadas N ora B acalá y Priscila Williems por el asesoramiento estadístico, y al Dr. Claudio Donoso y a los dos revisores anónimos por las sugerencias recibidas.

\section{REFERENCIAS}

AHLGREN, C.E. 1974. "Effects of fires on temperate forests: North Central United States. En: KOZL OWSKI, T y C. A HLGREN (eds.). Fire and Ecosystems. A cademic Press. New Y ork: 195-224.

BAILEY WILLIS, J. 1988. El Norte de la Patagonia. EUDEBA. Bs. A s., $500 \mathrm{pp}$

BARROS, V., V. CORDON, C. MOYANO, R. MENDEZ, J. FORQUERA y O.PIZZIO. 1983. Cartas de precipitación de la zona oeste de las provincias de Río N egro y Neuquén. Facultad de Cs. A grarias, Universidad Nacional del $\mathrm{Co}$ mahue/Centro Nac. Patagónico. Cinco Saltos.

BRUNO, J. 1982. Los incendios forestales en los Parques Nacionales. Estudio estadístico y análisis de incidencia. A dministración de Parques Nacionales. A rgentina, $45 \mathrm{pp}$

CABRERA, A. 1976. Regiones fitogeográficas argentinas. Enc. Arg. de A gric, y Jard. Tomo II Fase. 1. Ed. ACME S.A.C.I., 83 pp.

CONNELL, J.H y R.O. SLATYER. 1977. "M echanisms of succession in natural communities and their role in community stability and organization", Amm. Nat. 111: 1119. 1144.

DEZZOTTI, A. 1989. Distribución, estructura y dinámica de Austrocedrus chilensis. (D. Don) Florin et Boutleje (Cupressaceae) bajo distintas condiciones ecológicas. Tesis de Licenciatura en Cs. Biológicas. Bariloche, Universidad Nacional del Comahue, $74 \mathrm{pp}$.

DONOSO, C. 1981. Ecología Forestal. El bosque y su medio ambiente. Ed. Universitaria, Santiago.

K OZL OWSKI, T.T. y C.E. A HLGREN (eds.). 1974. Fire and ecosystems. A cademic Press, New Y ork, $542 \mathrm{pp}$.

LEBEDEFF, N. 1942. "Rejuvenecimiento del ciprés e influencia de la ganadería". Bol. For. 1938-40. Dir. Gral. Parques N acionales. Bs. A s.; 128-158.

LITTLE, S. 1974. "Effects of fires on temperate forests: Northeastern United States". En: KOZL OWSKI, T y C. A HLGREN (eds.). Fire and Ecosystems. A cademic Press, New Y ork: 225-250.

LOPEZ, J.A., J. DIAZ y C. LOPEZ. 1988. "Observaciones sobre regeneración natural de especies forestales autóctonas a un año del incendio forestal en el Parque Nacional Lago Puelo - Provincia del Chubut", Anales del VII Congreso Forestal Argentino. Santiago del Estero: 208-212.

HUECK, K. 1978. Los bosques de Sudamérica. Soc. Alemana de Cooperación Técnica, $474 \mathrm{pp}$

PICKETT, S.T.A., S.L. COLLINS y J.J. ARMESTO. 1987. "A hierarchical consideration of causes and mechanisms of succession", Vegetado 69: 109-114

ROME, W. 1980. Fire history terminology: Report of the ad hoc Committee. USDA-Forest Serv. Gral. Tech. Rep. R$\mathrm{M}-81.135-137$.

ROTHKU GEL, M. 1913. Los incendios en los Andes patagónicos. Dir. Gen. A gr. y Defensa A grícola. B ol. № 3, $22 \mathrm{pp}$.

ROTHKUGEL, M. 1916. Los bosques patagónicos. Min. de A gric, Bs. As., 204 pp.

ROVERE, A. 1991. Estudio experimental de la germinación y el desarrollo temprano del ciprés de la cordillera. (Austocedrus chilensis). Tesis de Licenciatura en Cs. Biológicas. Bariloche, Universidad Nacional del Comahue, $79 \mathrm{pp}$. 
SEIBERT, P. 1982. Carta de vegetación de la región de EI Bolsón y su aplicación a la planificación del uso de la tierra. Doc. Phytosociologica 2. Fund, para la Educ, la Cs. y la Cult. $120 \mathrm{pp}$

SOLBRIG, O. 1984. "Forests and W oodlands. The Southern A ndes and Sierras Pampeanas", Mountain Research and Development. 4(2): 163-173.
VEBLEN, T. y D. LORENZ. 1987. "Post-fire stand development of Austrocedrus chilensis forest in Northern Patagonia", Vegetatio 71: 113-126.

VEBLEN, T. y D. LORENZ. 1988. "Recent vegetation changes along the forest/steppe ecotone of Northern Patagonia", Annals Assoc. of Amer. Geogr. 78(1): 93111. 